\title{
Getting the Right Evidence After Drug Approval
}

\author{
Rick A. Vreman ${ }^{1,2 *}$, Hubert G. M. Leufkens ${ }^{1}$ and Aaron S. Kesselheim ${ }^{3}$ \\ ${ }^{1}$ Division of Pharmacoepidemiology and Clinical Pharmacology, Utrecht Institute for Pharmaceutical Sciences, Utrecht \\ University, Utrecht, Netherlands, ${ }^{2}$ National Health Care Institute (ZIN), Diemen, Netherlands, ${ }^{3}$ Program on Regulation, \\ Therapeutics and Law (PORTAL), Division of Pharmacoepidemiology and Pharmacoeconomics, Department of Medicine, \\ Brigham and Women's Hospital/Harvard Medical School, Boston, MA, United States
}

To generate comparative evidence in a timely fashion for drugs without restricting or delaying access, value-based pricing and reimbursement could be conditioned on a prospective, post-approval evidence generation plan.

Keywords: evidence, comparative evidence, regulation, pricing, value-based, health technology assessment, reimbursement

\section{OPEN ACCESS}

Edited by:

Maria Dimitrova,

Medical University-Sofia, Bulgaria

Reviewed by:

Domenico Criscuolo,

Italian Society of Pharmaceutical

Medicine, Italy

Prince Kasongo Mwila,

Potchefstroom Hospital, South Africa

${ }^{*}$ Correspondence:

Rick A. Vreman

r.a.vreman@uu.n

Specialty section:

This article was submitted to Pharmaceutical Medicine and Outcomes Research,

a section of the journal

Frontiers in Pharmacology

Received: 05 June 2020

Accepted: 14 August 2020

Published: 09 September 2020

Citation:

Vreman RA, Leufkens HGM and Kesselheim AS (2020) Getting the Right Evidence After Drug Approval.

Front. Pharmacol. 11:569535. doi: 10.3389/fphar.2020.569535

\section{INTRODUCTION}

Drug regulatory policies aim to balance evidence generation with patient access. Overly onerous demands for evidence lead to longer development times and higher costs. Approving drugs too early may lead to uninformed use due to unestablished or uncertain benefits and risks, potentially harming patients and adding to increased and unjustified health care spending (Eichler et al., 2018).

\section{DRUG APPROVAL VERSUS CLINICAL PRACTICE}

After a drug is approved, a key issue for patients, prescribers, and payers is how much clinical benefit on patient-relevant outcomes can be expected compared to available alternatives. However, formal regulatory requirements by the FDA or EMA do not include active control comparisons based on patient-relevant clinical endpoints, resulting in a limited number of regulatory dossiers containing such data (Kim and Prasad, 2015; Hatswell et al., 2016; Vivot et al., 2017; Morant and Vestergaard, 2018; Chen et al., 2019; Naci et al., 2019). Furthermore, drugs may have variable comparative effects in different subgroups of the patient population, which often remain unstudied or not captured in the label.

A lack of comparative evidence may be particularly evident for drugs approved via expedited pathways such as accelerated approval in the United States or conditional approval in Europe. Both regulatory systems require under such circumstances additional evidence generation post-approval for these drugs, but studies have revealed that these post-approval trials often have similar characteristics to pre-approval trials, and are too frequently delayed or difficult to get finished (Hoekman et al., 2016; Naci et al., 2017; Bloem et al., 2019). After drugs are approved and reimbursed, manufacturers have little incentive to perform additional active control trials within the same indication. Indeed, $81 \%$ of industry initiated non-obligated post-approval trials were to study new indications or indication expansions rather than approved indications (Skydel et al., 2019).

Lack of comparative evidence can complicate treatments decisions. For example, in chronic lymphocytic leukemia, treatment choices can be based on genetic abnormalities, fitness of patients, 
previous treatments and timing of relapse, among other factors. The myriad of newly approved targeted treatment options provide a promising outlook for patients with this condition (Yeung and Shadman, 2019). However, a recent review of available treatment options for chronic lymphocytic leukemia also emphasizes that the current available evidence leaves many questions unanswered because of single-arm trial designs and a lack of robust data on active comparisons, on combination regimens, on long-term outcomes, and on treatment effects in subpopulations (Yeung and Shadman, 2019).

\section{WHY ARE THERE SO MANY UNANSWERED QUESTIONS?}

It is perhaps unsurprising that current regulatory standards lead to many remaining unanswered questions at the time of drug approval. A positive benefit-risk balance as assessed by regulators does not necessarily provide answers to questions on added therapeutic benefit that may be of most interest to clinicians and payers (Eichler et al., 2019). Additionally, indications can be granted on average effects without sufficient power to draw conclusions for subpopulations.

If questions about comparative clinical effects in relevant subgroups at approval are only partly addressed by postapproval trials obligated by regulators, how can we get the evidence patients, clinicians and payers need?

One pathway may be to leverage organizations that conduct health technology assessment (HTA), a term that encompasses clinical and economic value assessments of new drugs informed by the evidence and medical experts. Independent organizations in the United States such as the Institute for Clinical and Economic Review (ICER) systematically assess comparative benefits on patient-relevant endpoints of certain drugs. ICER explicitly highlights remaining uncertainties related to the available evidence beyond the benefit-risk balance. In Europe, formal HTA processes exist in most countries, leading to dossiers about comparative benefit and economic value of medicines similar to those of ICER. However, ICER has no mandate to enforce evidence generation and in Europe additional evidence generation for HTA purposes is organized nationally with only little international coordination.

Besides outlining the remaining uncertainties and the types of trials needed to resolve them, HTA processes can also provide suggestions for reasonable drug prices based on the drug's effects and the uncertainties around these effects. Linking the value that a product delivers to its price may incentivize the development of transformative drugs. Value-based pricing mechanisms have contributed to lower drug prices in Europe as compared to the United States (Kang et al., 2020). However, lowering prices for drugs for which clinically relevant comparative benefits are still unclear does not solve the problem that patients and clinicians face when they must decide which treatment to start. Ultimately, it must be established whether a drug provides meaningful benefits over alternative treatment options.

Clearly, there are institutes such as HTA organizations that have the capacity to outline the most relevant uncertainties to patients, clinicians, and payers that should be resolved postapproval. But who can enforce this type of evidence generation?

\section{PROMOTING POST-APPROVAL EVIDENCE GENERATION}

To increase post-approval evidence generation, regulators could be given more authority to work with HTA bodies to determine an agenda for post-approval comparative trials related to newly-approved drugs. In a comprehensive review of regulatory and HTA dossiers, we found that regulators and HTA bodies were usually able to agree on appropriate endpoints and comparators in pre-approval scientific advice procedures, and that manufacturers often followed this combined advice for clinical trials (Tafuri et al., 2016). Such joint efforts do not yet exist for post-approval trials, and through this pathway, remaining uncertainties related to comparative clinical effects within the relevant patient populations can be identified at approval.

However, studies have pointed to regulators' inability to consistently enforce post-approval trial obligations, because it is politically and socially challenging to withdraw marketing authorization purely because evidence generation is delayed. In a previous study, seven guiding principles were put forward to guide the generation of evidence post-approval. One of the principles states that it is necessary to create the right incentives for generating comparative evidence post-approval (Cipriani et al., 2020). One way to do this would be to make drug prices conditional upon resolving the most important uncertainties, leading to price premiums for more certainty and price penalties for delayed evidence generation. Such a prospective approach can facilitate fair return on investment for post-approval evidence generation for manufacturers while simultaneously reducing financial uncertainty for payers.

The viability of such an approach depends on the willingness of the involved stakeholders. In the US, private insurers already negotiate drug prices and they have stressed the relevance of value assessments for coverage and pricing decisions (Augustovski and McClellan, 2019). However, there is no governmental HTA institute and there is also no formal coordination between the FDA and nongovernmental HTA institutes. In Europe, results of coverage with evidence development schemes are mixed and a central approach that is aligned with the EMA is lacking. The European Network for HTA (EUnetHTA) may facilitate the coordination between the EMA and national HTA institutes. Most vital is that price incentives facilitate research post-approval that is not duplicative, answers the most important uncertainties for all stakeholders, and is done in the most efficient and timely way.

\section{CONCLUSION}

Clinical evidence at drug approval is often not sufficient to ensure informed clinical and reimbursement decision making. One 
solution to incentivize companies to generate such comparative evidence in a timely fashion without restricting or delaying access could be to condition value-based pricing and reimbursement on a prospective, post-approval evidence generation plan. Whether such schemes will deliver on their promise depends on the quality of early dialogue between stakeholders, predictability of the outcomes, and on stakeholders' ability to establish a fair balance between sufficient prospects of returns on trial investments for manufacturers and reasonable value for money for payers.

\section{AUTHOR'S NOTE}

HGML declares that he is a member of the Lygature Leadership Team.

\section{REFERENCES}

Augustovski, F., and McClellan, M. B. (2019). Current Policy and Practice for Value-Based Pricing. Value Health 22 (6), S4-S6. doi: 10.1016/j.jval.2019.04.1918

Bloem, L. T., Mantel-Teeuwisse, A. K., Leufkens, H. G. M., De Bruin, M. L., Klungel, O. H., and Hoekman, J. (2019). Postauthorization Changes to Specific Obligations of Conditionally Authorized Medicines in the European Union: A Retrospective Cohort Study. Clin. Pharmacol. Ther. 105 (2), 426-435. doi: 10.1002/cpt.1169

Chen, E. Y., Raghunathan, V., and Prasad, V. (2019). An Overview of Cancer Drugs Approved by the US Food and Drug Administration Based on the Surrogate End Point of Response Rate. JAMA Intern. Med. 179 (7), 915-921. doi: 10.1001/jamainternmed.2019.0583

Cipriani, A., Ioannidis, J. P. A., Rothwell, P. M., Glasziou, P., Li, T., Hernandez, A. F., et al. (2020). Generating comparative evidence on new drugs and devices after approval. Lancet 395 (10228), 998-1010. doi: 10.1016/S0140-6736(19)33177-0

Eichler, H.-G., Barker, R., Bedlington, N., Bouvy, J. C., Broekmans, A. W., Bucsics, A., et al. (2018). The evolution of adaptiveness: balancing speed and evidence. Nat. Rev. Drug Discovery 17 (12), 845-846. doi: 10.1038/nrd.2018.90

Eichler, H.-G., Enzmann, H., and Rasi, G. (2019). Added therapeutic benefit and drug licensing. Nat. Rev. Drug Discovery 18 (9), 651-652. doi: 10.1038/d41573019-00068-x

Hatswell, A. J., Baio, G., Berlin, J. A., Irs, A., and Freemantle, N. (2016). Regulatory approval of pharmaceuticals without a randomised controlled study: analysis of EMA and FDA approvals 1999-2014. BMJ Open 6 (6), e011666. doi: 10.1136/bmjopen-2016-011666

Hoekman, J., Klamer, T. T., Mantel-Teeuwisse, A. K., Leufkens, H. G. M., and De Bruin, M. L. (2016). Characteristics and follow-up of postmarketing studies of conditionally authorized medicines in the EU. Br. J. Clin. Pharmacol. 82 (1), 213-226. doi: 10.1111/bcp.12940

Kang, S.-Y., Bai, G., DiStefano, M. J., Socal, M. P., Yehia, F., and Anderson, G. F. (2020). Comparative Approaches to Drug Pricing. Annu. Rev. Public Health 41, 499-512. doi: 10.1146/annurev-publhealth-040119-094305

Kim, C., and Prasad, V. (2015). Cancer Drugs Approved on the Basis of a Surrogate End Point and Subsequent Overall Survival: An Analysis of 5 Years of US Food and Drug Administration Approvals. JAMA Intern. Med. 175 (12), 1992-1994. doi: 10.1001/jamainternmed.2015.5868

Morant, A. V., and Vestergaard, H. T. (2018). European Marketing Authorizations Granted Based on a Single Pivotal Clinical Trial: The Rule or the Exception? Clin. Pharmacol. Ther. 104 (1), 169-177. doi: 10.1002/cpt.900

\section{AUTHOR CONTRIBUTIONS}

All authors contributed to the article and approved the submitted version.

\section{FUNDING}

HL declares that he is a member of the Lygature Leadership Team. AK's work is supported by Arnold Ventures, as well as the Harvard-MIT Center for Regulatory Science. The funders were not involved in the study design, collection, analysis, interpretation of data, the writing of this article or the decision to submit it for publication.

Naci, H., Smalley, K. R., and Kesselheim, A. S. (2017). Characteristics of Preapproval and Postapproval Studies for Drugs Granted Accelerated Approval by the US Food and Drug Administration. JAMA 318 (7), 626636. doi: 10.1001/jama.2017.9415

Naci, H., Davis, C., Savović, J., Higgins, J. P. T., Sterne, J. A. C., Gyawali, B., et al. (2019). Design characteristics, risk of bias, and reporting of randomised controlled trials supporting approvals of cancer drugs by European Medicines Agency, 2014-16: cross sectional analysis. BMJ 366, 15221. doi: 10.1136/bmj.15221

Skydel, J. J., Luxkaranayagam, A. T., Dhruva, S. S., Ross, J. S., and Wallach, J. D. (2019). Analysis of Postapproval Clinical Trials of Therapeutics Approved by the US Food and Drug Administration Without Clinical Postmarketing Requirements or Commitments. JAMA Netw. Open 2 (5), e193410-e193410. doi: 10.1001/jamanetworkopen.2019.3410

Tafuri, G., Pagnini, M., Moseley, J., Massari, M., Petavy, F., Behring, A., et al. (2016). How aligned are the perspectives of EU regulators and HTA bodies? A comparative analysis of regulatory-HTA parallel scientific advice. Br. J. Clin. Pharmacol. 82 (4), 965-973. doi: 10.1111/bcp.13023

Vivot, A., Jacot, J., Zeitoun, J.-D., Ravaud, P., Crequit, P., and Porcher, R. (2017). Clinical benefit, price and approval characteristics of FDA-approved new drugs for treating advanced solid cancer, 2000-2015. Ann. Oncol. 28 (5), 1111-1116. doi: 10.1093/annonc/mdx053

Yeung, C. C. S., and Shadman, M. (2019). How to Choose the Best Treatment and Testing for Chronic Lymphocytic Leukemia in the Tsunami of New Treatment Options. Curr. Oncol. Rep. 21 (8), 74. doi: 10.1007/s11912-019-0819-x

Disclaimer: The views expressed in this article are the personal views of the authors and may not be understood or quoted as being made on behalf of or reflecting the position of the agencies or organizations with which the authors are affiliated.

Conflict of Interest: The authors declare that the research was conducted in the absence of any commercial or financial relationships that could be construed as a potential conflict of interest.

Copyright (c) 2020 Vreman, Leufkens and Kesselheim. This is an open-access article distributed under the terms of the Creative Commons Attribution License (CC BY). The use, distribution or reproduction in other forums is permitted, provided the original author(s) and the copyright owner(s) are credited and that the original publication in this journal is cited, in accordance with accepted academic practice. No use, distribution or reproduction is permitted which does not comply with these terms. 